\title{
Differential roles of the fan-shaped body and the ellipsoid body in Drosophila visual pattern memory
}

\author{
Yufeng Pan, ${ }^{1,2}$ Yanqiong Zhou, ${ }^{1,2}$ Chao Guo, ${ }^{1,2}$ Haiyun Gong, ${ }^{1}$ Zhefeng Gong, ${ }^{1,3}$ \\ and Li Liu ${ }^{1,3}$ \\ ${ }^{1}$ State Key Laboratory of Brain and Cognitive Science, Institute of Biophysics, Chinese Academy of Sciences, Chaoyang District, \\ Beijing 100101, People's Republic of China; ${ }^{2}$ Graduate University of Chinese Academy of Sciences, Beijing 100039, People's \\ Republic of China
}

\begin{abstract}
The central complex is a prominent structure in the Drosophila brain. Visual learning experiments in the flight simulator, with flies with genetically altered brains, revealed that two groups of horizontal neurons in one of its substructures, the fan-shaped body, were required for Drosophila visual pattern memory. However, little is known about the role of other components of the central complex for visual pattern memory. Here we show that a small set of neurons in the ellipsoid body, which is another substructure of the central complex and connected to the fan-shaped body, is also required for visual pattern memory. Localized expression of rutabaga adenylyl cyclase in either the fan-shaped body or the ellipsoid body is sufficient to rescue the memory defect of the rut ${ }^{2080}$ mutant. We then performed RNA interference of rutabaga in either structure and found that they both were required for visual pattern memory. Additionally, we tested the above rescued flies under several visual pattern parameters, such as size, contour orientation, and vertical compactness, and revealed differential roles of the fan-shaped body and the ellipsoid body for visual pattern memory. Our study defines a complex neural circuit in the central complex for Drosophila visual pattern memory.
\end{abstract}

The central complex is a prominent structure in the central brain of adult insects. Numerous studies have indicated that it is an important center for higher-order brain functions including locomotor control (Strauss and Heisenberg 1993; Martin et al. 1999; Strauss 2002; Poeck et al. 2008), processing of $E$ vector orientation information (Vitzthum et al. 2002; Heinze and Homberg 2007), courtship behavior (Popov et al. 2005; Sakai and Kitamoto 2006), olfactory long-term memory (Wu et al. 2007), visual pattern memory (Liu et al. 2006; Wang et al. 2008), and spatial orientation memory (Neuser et al. 2008).

In Drosophila melanogaster, the central complex consists of four interconnected substructures: the protocerebral bridge $(\mathrm{pb})$, the fan-shaped body (fb), the ellipsoid body (eb), and the paired noduli (no) (Hanesch et al. 1989; Renn et al. 1999). The central complex has been found to be involved in multiple forms of learning and memory. Memory for two visual pattern parameters in tethered flight requires rutabaga (rut) function in two distinct large field neurons (F neurons) in the fan-shaped body (Liu et al. 2006). The central complex is not involved in short-term olfactory memory, but a subset of large field neurons in the ellipsoid body that are called "R neurons" (R2/R4m neurons) participate in the NMDA receptor-dependent long-term memory consolidation (Wu et al. 2007). Another group of R neurons (R3/R4d) are necessary for spatial orientation memory, which is similar to the working memory in vertebrates (Neuser et al. 2008).

Concerning Drosophila visual pattern memory in tethered flight, large field neurons in the first layer (F1 neurons) and fifth layer (F5 neurons) of the fan-shaped body are, respectively, responsible for rut-dependent memory for "contour orientation" and "elevation" (Liu et al. 2006), which are two out of five known learnable pattern parameters (Ernst and Heisenberg 1999; Tang

\footnotetext{
${ }^{3}$ Corresponding authors.

E-mail liuli@sun5.ibp.ac.cn; fax 86-10-64853625.

E-mail zfgong@moon.ibp.ac.cn; fax 86-10-64853625.

Article is online at http://www.learnmem.org/cgi/doi/10.1101//m.1331809.
}

and Guo 2001). Meanwhile, protein kinase G (PKG) was found to be required in the ellipsoid body for visual pattern memory (Wang et al. 2008). Memory for "contour orientation" and "elevation" both needed PKG function in a subset of the ellipsoid body large field neurons. However, the functional relationship between the fan-shaped body and ellipsoid body in visual pattern memory is not well understood.

To address whether the fan-shaped body and ellipsoid body work together or function redundantly in Drosophila visual pattern memory, we performed rut rescue experiments by targeted expression of wild-type rut cDNA in rut mutant flies, and knocked down rut in the central complex by RNA interference. We found that both the fan-shaped body and ellipsoid body were involved in visual pattern memory. As for the four learnable pattern parameters: "contour orientation," "elevation," "size," and "vertical compactness" (Ernst and Heisenberg 1999), F1 and F5 neurons were required for memory for "contour orientation" and "elevation," respectively, while a subset of R neurons (R2/ $\mathrm{R} 4 \mathrm{~m}$ ) seemed to be involved in memory for all the parameters in a parameter-independent manner. Our results indicated that the fan-shaped body and ellipsoid body neurons might be both required, but in different ways, for Drosophila visual pattern memory.

\section{Results}

Overexpression of rut cDNA in either the fan-shaped body or ellipsoid body neurons was sufficient to restore rut $^{2080}$ visual pattern memory defect

In Drosophila, visual pattern memory has been studied in detail in tethered flight (Fig. 1A; Wolf and Heisenberg 1991; Brembs and Heisenberg 2000). It was previously found that rut ${ }^{2080}$, a P-element insertion allele, was defective in visual pattern memory (Fig. 1C; Heisenberg et al. 2001; Liu et al. 2006). We successfully rescued visual pattern memory in the rut ${ }^{2080}$ mutant flies with restricted 
A

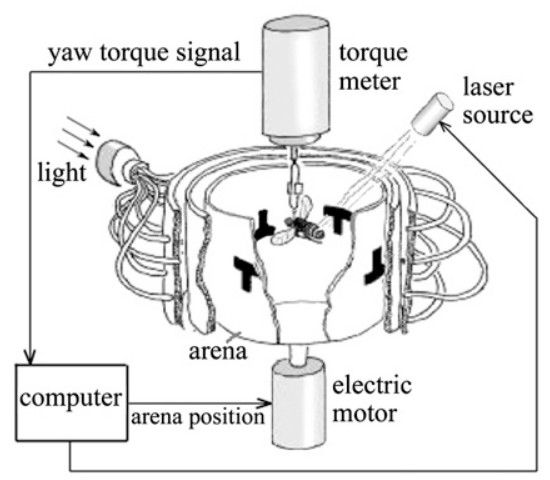

B

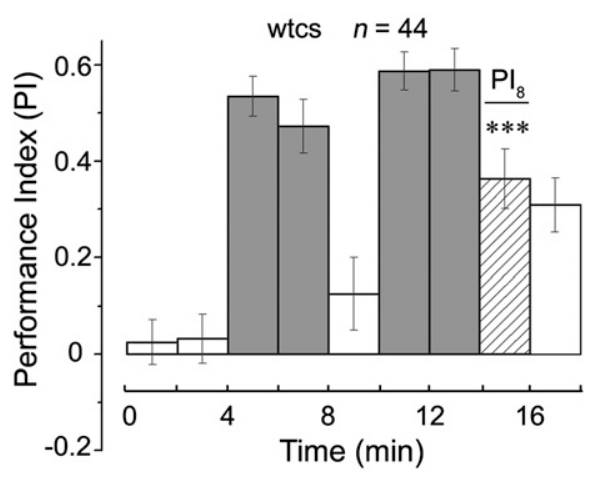

C

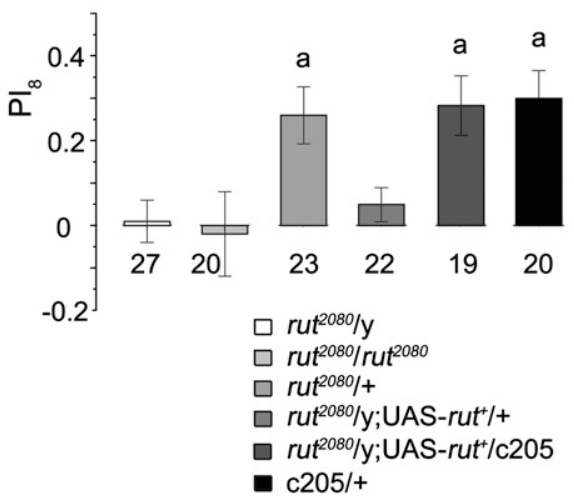

Figure 1. Visual pattern memory in the wild-type and rut ${ }^{2080}$ mutant flies in the flight simulator. (A) Schematic diagram of the flight simulator. (B) Performance of wild-type Canton S flies in an 18-min procedure $(n=$ 44). Bars show performance indices (PIs) of nine consecutive 2-min periods including pretest (open bars, $\mathrm{Pl}_{1}$ and $\mathrm{Pl}_{2}$ ), training (dark gray bars, $\mathrm{Pl}_{3}, \mathrm{Pl}_{4}, \mathrm{Pl}_{6}$, and $\mathrm{PI}_{7}$ ), and memory test (open bars, $\mathrm{PI}_{5}$ and $\mathrm{PI}_{9}$; hatched bars, $\mathrm{Pl}_{8}$.. $\mathrm{Pl}_{8}$ is used as an index of 2-min short-term memory throughout. T patterns were used as visual stimuli for pattern parameter "elevation." (C) Overexpression of rut cDNA targeted by c205 could restore the memory defect of rut ${ }^{2080}$ flies. Numbers below bars indicate numbers of flies. $\left.{ }^{* \star *}\right) P<0.001$ compared with the chance level zero (one-sample $t$-test); (a) $P<0.01$ in comparison with zero (one-sample $t$-test) and $P>0.05$ in comparison with wild type (two independent samples $t$-test). Error bars are SEMs throughout.

expression of rut cDNA using the UAS/GAL4 system (Brand and Perrimon 1993). Control flies carrying rut ${ }^{2080}$ and a copy of UAS$r u t^{+}$but no GAL4 driver $\left(r u t^{2080} / \mathrm{y}\right.$; UAS-rut $\left.t^{+}++\right)$were compared with those bearing GAL4 drivers. Introduction of UAS-rut ${ }^{+}$did not increase the memory index of the $r u t^{2080}$ flies (Fig. 1C). According to Liu et al. (2006), in a total of 27 driver lines expressing GAL4 in different neuropil regions, there were seven lines with an expression in the F5 neurons in which the memory for "elevation" was restored.

As we recently found that PKG was required in a subset of the ellipsoid body neurons (R2/R4m) for visual pattern memory (Wang et al. 2008), we asked whether Rut also was required there. Indeed, when using $\mathrm{T}$ patterns as conditioned stimuli, $r u t^{2080}$ flies expressing rut $t^{+}$ectopically in the R2/R4m neurons (c819) (Fig. 2C) showed significantly higher memory levels than control flies $(t=$ $2.41, P<0.05$; Fig. 2A). In contrast, expression of $r u t^{+}$in the R3/ R4d neurons (c232) (Fig. 2C) failed to restore the rut ${ }^{2080}$ memory defect ( $t=0.14, P=$ NS [not significant]; Fig. 2A). These results indicated that the $\mathrm{R} 2 / \mathrm{R} 4 \mathrm{~m}$ neurons might also be required for visual pattern memory for "elevation," like the F5 neurons in the fan-shaped body. To confirm this, we targeted rut ${ }^{+}$expression using two other GAL4 lines, c42 and c547, which also labeled the R2/R4m neurons (Fig. 2C). Consistent with the rescue results with c819, rut $^{+}$expression driven by c42 or c547 could successfully restore the rut ${ }^{2080}$ memory defect $(t=4.13, P<0.001$ and $t=3.37$, $P<0.01$, respectively; Fig. $2 \mathrm{~A})$. Taking these results together, we concluded that aside from the F5 neurons in the fan-shaped body, the R2/R4m neurons in the ellipsoid body could independently restore the rut ${ }^{2080}$ memory defect when using $\mathrm{T}$ patterns as conditioned stimuli.

\section{Adult-restricted expression of Rut was sufficient to rescue rut ${ }^{2080}$ memory defect}

To exclude potential developmental defects, we used tub-GAL80 ${ }^{\text {ts }}$ to suppress GAL4 function in the R2/R4m neurons during development. Flies with induced expression of rut for $12 \mathrm{~h}$ at $30^{\circ} \mathrm{C}$ in the R2/R4m neurons ( $r u t^{2080} / \mathrm{y} ;$ tub-GAL80 $/$ ts $/$; UAS-rut $t^{+} / \mathrm{c} 819$ ) had significantly higher memory levels compared with those without induced expression of $r u t^{+}(t=2.65, P<0.05$; Fig. 2B). Furthermore, these flies showed significantly better memory than those without GAL4 driver ( rut $^{2080} / \mathrm{y} ; \mathrm{tub}-\mathrm{GAL} 80^{\mathrm{ts}} /+$; UAS-rut $\left.t^{+} /+\right)$ that had also been heat shocked for $12 \mathrm{~h}(t=2.97, P<0.01$; Fig. $2 \mathrm{~B})$. These results demonstrated that acute Rut function in the adult $\mathrm{R} 2 / \mathrm{R} 4 \mathrm{~m}$ neurons was sufficient for visual pattern memory.

\section{RNAi knockdown of rut in either the fan-shaped body or} ellipsoid body neurons abolished visual pattern memory As indicated above, independent expression of $r^{+} t^{+}$in the F5 or R2/ $\mathrm{R} 4 \mathrm{~m}$ neurons could both restore visual pattern memory for "elevation" of the rut ${ }^{2080}$ mutant. This suggests that Rut function in either of these two neuronal subtypes was sufficient for normal visual pattern memory. To test if they were functionally interchangeable, we performed RNA interference (RNAi) in either the fan-shaped body or ellipsoid body neurons. Flies with rut RNAi expression in the F5 neurons, labeled by c205, showed significantly impaired visual pattern memory compared with control flies $\left(t=3.12, P<0.01\right.$ against the UAS-rut $t^{\mathrm{RNAi}} /+$ flies; $t=2.79, P<$ 0.01 against the $\mathrm{c} 205 /+$ flies), so did those with rut RNAi driven by c819 $\left(t=3.02, P<0.01\right.$ against the UAS-rut $t^{\mathrm{RNAi}} /+$ flies; $t=2.72, P<$ 0.05 against the $c 819 /+$ flies; Fig. 3A). Meanwhile, RNAi-mediated knockdown of rut driven by c232 did not affect visual pattern memory $\left(t=0.58, P=\right.$ NS compared with the UAS-rut $t^{\mathrm{RNAi}} /+$ flies; $t=$ $0.54, P=$ NS compared with the c232/+ flies; Fig. 3A). Hence, we concluded that the F5 (c205) and R2/R4m (c819) neurons were both necessary for visual pattern memory.

\section{A critical role of rut mutant allele}

The RNAi result is not consistent with the rescue experiments described above. If both neurons ( $\mathrm{F} 5$ and R2/R4m) were necessary for the constitution of visual pattern memory for "elevation," then 
A
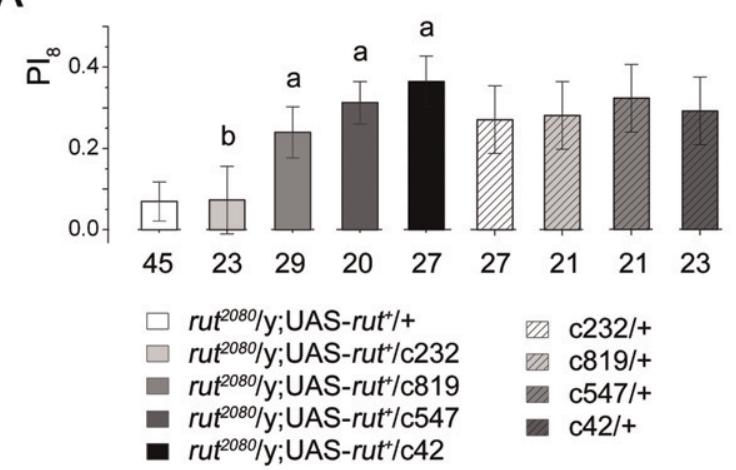

C
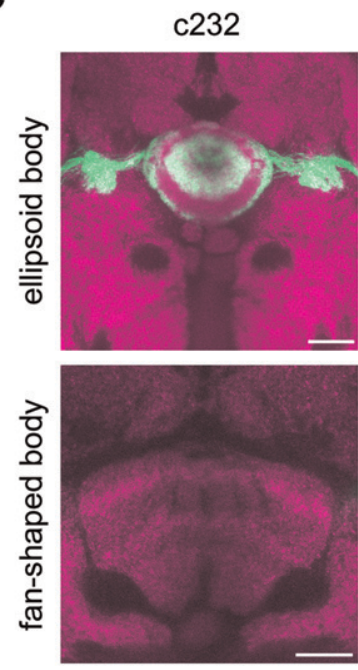

B

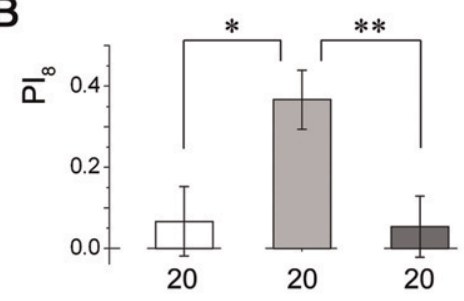

rut $^{2080} / \mathrm{y} ;$ GAL80/+;UAS-rut $/ \mathrm{c819}$

rut $^{2080} / \mathrm{y} ; \mathrm{GAL} 80 /+;$ UAS-rut $/ \mathrm{c} 819$

rut ${ }^{2080 / y}$;GAL80/+;UAS-rut $/++$ $19^{\circ} \mathrm{C}$

$30^{\circ} \mathrm{C}$

Figure 2. Overexpression of rut $\mathrm{CDNA}$ in the R2/R4m neurons was sufficient to rescue rut ${ }^{2080}$ visual pattern memory defect. $(A) c 819, \mathrm{c} 547$, and $c 42$ could fully restore visual pattern memory for "elevation" by driving rut $t^{+}$expression, but c232 could not. (B) Flies with genotype rut ${ }^{2080} / \mathrm{y} ; \mathrm{tub}-\mathrm{GAL} 80^{\text {ts }} /$ $+; \mathrm{UAS}-$ rut $^{+} / \mathrm{c} 819$ showed no memory if always kept at $19^{\circ} \mathrm{C}$, but they showed significantly higher memory if transferred to $30^{\circ} \mathrm{C} 12 \mathrm{~h}$ before behavioral experiments. This acute rescue effect was not observed in the flies without GAL4 driver (rut $\left.t^{2080} / y ; t u b-G A L 80^{t s} /+; U A S-r u t^{+} /+\right)$. (C) Expression patterns of c232, c819, c547, and c42 in the fan-shaped body and the ellipsoid body are shown by green fluorescent protein (GFP) (green). The synaptic neuropil is stained by nc82 antibody (magenta). c232 labels the R3/R4d neurons, while the other three drivers label the R2/R4m neurons. Only c42 has expression in the first layer of the fan-shaped body (F1 neurons). Numbers below bars indicate numbers of flies. (a) $P<0.05$ compared with flies with genotype rut ${ }^{2080} /$ y; UAS-rut ${ }^{+} /+$and $P>0.05$ compared with corresponding GAL4 controls. (b) $P>0.05$ compared with rut ${ }^{2080} / \mathrm{y} ;$ UAS-rut $t^{+} /+$flies. $\left(^{*}\right) P<0.05 ;\left({ }^{* *}\right) P<0.01$. All comparisons were made using two independent samples $t$-test. Error bars indicate SEM. Scale bars are $20 \mu \mathrm{m}$ for both. The scale bars in the first column apply to all panels in the same row.

for only one of them the rescue should be expected to be unsuccessful. However, this was not the case: In both neurons the memory was successfully restored by expression of $r u t^{+}$. One possible explanation could be that there exists residual rut function in the fan-shaped body or ellipsoid body of rut ${ }^{2080}$ flies. To compare the rut expression levels in the rut $t^{2080}$ mutant and RNAiexpressing flies, we performed quantitative polymerase chain reaction (PCR) and found that the rut mRNA level in the rut ${ }^{2080}$ flies was significantly lower than in the wild-type flies, but indeed significantly higher than in the rut RNAi flies (Fig. 3B).

The fact that rut ${ }^{2080}$ is a hypomorph implies that the independent rescue in only the F5 or R2/R4m neurons might not be successful in a rut null mutant. Among the rut mutants, rut ${ }^{1}$ was generated by EMS (ethyl methane sulfonate) mutagenesis, which caused a point mutation in a conserved site and abolished Rut activity (Dudai and Zvi 1984; Livingstone et al. 1984; Han et al. 1992; Levin et al. 1992). Hemizygous male rut ${ }^{1}$ mutant flies with one copy of UAS-rut ${ }^{+}$showed a significant visual pattern memory defect, while heterozygous rut $t^{1}$ female flies with one copy of UAS- $r u t^{+}$showed normal visual pattern memory (Fig. 4A). Pan-neuronal expression of rut cDNA with elav-GAL4 rescued the rut ${ }^{1}$ memory defect $(t=2.47, P<0.05)$, while targeted expression of $r u t^{+}$ in neither the F5 neurons (c205: $t=0.79, P=\mathrm{NS}$; 104y: $t=1.21$, $P=\mathrm{NS}$ ) nor R2/R4m neurons (c819: $t=1.15, P=\mathrm{NS}$ ) could restore the memory defect (Fig. 4A). However, overexpression of $r u t^{+}$in both the F5 and R2/R4m neurons (104y + c819; Fig. 4B) significantly improved the visual pattern memory level $(t=2.1, P<0.05$; Fig. 4A). These results indicated that the combination of the F5 and R2/R4m neurons, but neither of them alone, were sufficient for visual pattern memory in the rut ${ }^{1}$ mutant.

\section{Functional differentiation of the fan-shaped body and ellipsoid body neurons}

Since the fan-shaped body and ellipsoid body neurons are both required for visual pattern memory, they may work together but function differentially. Liu et al. (2006) indicated that the F1 neurons were specific for memory for the parameter "contour orientation" and F5 for "elevation," suggesting that the F neurons 
A

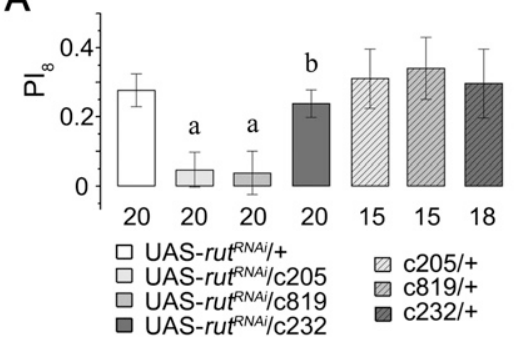

Figure 3. Targeted RNA interference of rut could impair visual pattern memory. (A) RNAi silencing of rut in either the F5 (c205) or R2/R4m (c819) neurons abolished visual pattern memory for "elevation," while knockdown of rut in the R3/R4d (c232) neurons had no effect on visual pattern memory. (B) rut mRNA level in the RNAi silencing flies was significantly lower than in the wild-type and rut ${ }^{2080}$ mutant flies $(n=4$ for all). Numbers below bars indicate numbers of flies. (a) $P<0.05$ compared with the UAS-rut ${ }^{R N A i} /+$ flies or with corresponding GAL4 controls; (b) $P>$ 0.05 compared with the UAS-rut ${ }^{R N A i} /+$ or $\mathrm{c} 232 /+$ flies (two independent samples $t$-test). ( $\left.{ }^{*}\right) P<0.05 ;\left({ }^{* * *}\right) P<0.001$ (ANOVA). Error bars indicate SEM.

might participate in processing specific visual pattern information. So we turned to use other pattern parameters such as size and vertical compactness, which could be discriminated and memorized by the wild-type but not the rut ${ }^{2080}$ mutant flies (Fig. 5A). Overexpression of $\mathrm{rut}^{+}$in the F5 neurons (c205) fully rescued visual pattern memory for "elevation" but none of the other three pattern parameters $(t=3.07, P<0.01$ for "elevation"; $t=0.47, P=$ NS for "contour orientation"; $t=0.46, P=$ NS for "size"; $t=0.34, P=$ NS for "vertical compactness"), while overexpression of $\mathrm{rut}^{+}$in the F1 neurons driven by NP6510 could only restore visual pattern memory for "contour orientation" $(t=3.35, P<0.01$ for "contour orientation"; $t=0.09, P=$ NS for "elevation"; $t=0.52, P=$ NS for "size"; $t=0.55$, $P=$ NS for "vertical compactness"; Fig. $5 \mathrm{~B})$. However, overexpression of $\mathrm{rut}^{+}$in the $\mathrm{R} 2 / \mathrm{R} 4 \mathrm{~m}$ neurons driven by c819 or c42 could restore visual pattern memory for all four parameters (c819: $t=3.59, P<$ 0.001 for "elevation"; $t=3.23, P<0.01$ for "contour orientation"; $t=3.36, P<0.01$ for "size"; $t=2.89, P<0.01$ for "vertical compactness"; c42: $t=2.54, P<0.05$ for "elevation"; $t=4.46, P<0.001$ for "contour orientation"; $t=2.33, P<0.05$ for "size"; $t=2.76, P<0.01$ for "vertical compactness"; Fig. 5C). These results indicated that the $\mathrm{F}$ neurons (F1 and F5) might participate in visual pattern memory in a parameter-dependent manner, whereas the R2/R4m neurons might do so in a parameter-independent manner. Thus, the $\mathrm{F}$ and $\mathrm{R}$ neurons might function differentially for visual pattern memory.

\section{Discussion}

In this study, we reported that a subset of the ellipsoid body neurons were also necessary for Rut-dependent visual pattern memory, in addition to the previously described horizontal neurons in the

B

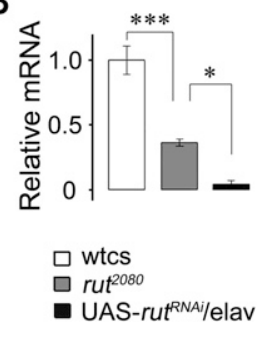

$\mathbf{A}$

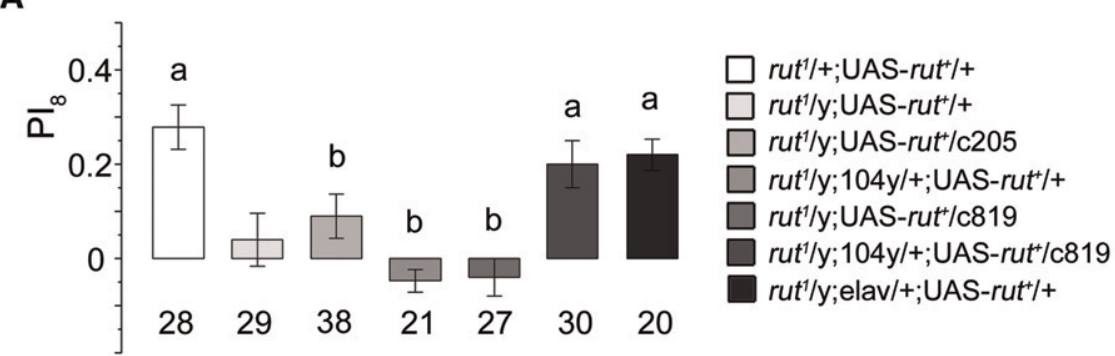

B
$104 y$

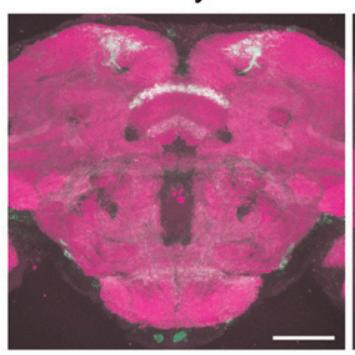

fan-shaped body (Liu et al. 2006). We demonstrated that these substructures of the central complex play different roles in visual pattern memory. Moreover, our experiments revealed that the choice of mutant allele was crucial when using the rutabaga rescue strategy.

\section{Necessary and sufficient structures of the central complex for visual pattern memory}

To localize the physical correlates of memory in the Drosophila central nervous system, one usually utilizes two distinct ways: (1) functional knockdown of "memory genes" or neural transmission in specific brain regions (Connolly et al. 1996; Dubnau et al. 2001; McGuire et al. 2001; Liu et al. 2006), and (2) functional rescue by targeted expression of a "memory gene" in the respective mutant (Zars et al. 2000a,b; Akalal et al. 2006; Liu et al. 2006; Thum et al. 2007). The former defines a structure necessary for memory formation, while the latter identifies a structure that is sufficient. rutabaga is such a gene that is involved in many forms of learning and memory in Drosophila. Functional rescue of the rut ${ }^{2080}$ mutant in olfactory aversive learning, olfactory reward learning, spatial learning, and visual pattern memory in tethered flight revealed distinct brain structures for memory formation: mushroom bodies for aversive olfactory learning (Zars et al. 2000a; Akalal et al. 2006), projection neurons or mushroom bodies for olfactory reward learning (Thum et al. 2007), median bundle for spatial learning (Zars et al. 2000b), and fan-shaped body for visual pattern memory (Liu et al. 2006). It should be noted that all these rescue experiments were done in the rut ${ }^{2080}$ mutant. The mutation is caused by a $P$-element insertion $155 \mathrm{bp}$ upstream of the rut gene (Han et al. 1992), which leads to a reduced rut level (Fig. 3B). The results of 
our rescue experiments demonstrate that using a hypomorphic mutant allele is not optimal for determining the sufficiency of a brain region for a given task. For example, although restoring Rut function in the fan-shaped body rescued visual pattern memory successfully, this cannot exclude the involvement of other regions owing to the residual Rut activity in the $r u t^{2080}$ flies. Therefore, it is crucial to perform rescue experiments in a null mutant. rut ${ }^{1}$ appears to be a suitable candidate, as the point mutation in the gene leads to a complete loss of Rut activity in both cultured cells (Levin et al. 1992) and head homogenate extracts (Dudai and Zvi 1984; Livingstone et al. 1984; Han et al. 1992). Overexpression of $r u t^{+}$in either the F5 or R2/R4m neurons alone in the rut ${ }^{1}$ mutant failed to restore visual pattern memory, implying that neither the fan-shaped body nor ellipsoid body neurons were sufficient. However, a combination of these two regions did succeed in rescuing the rut ${ }^{1}$ memory defect. Taken together with our RNAi results that indicated necessary roles of both the F5 and R2/R4m neurons, it could be concluded that these fan-shaped body and ellipsoid body neurons seemed to be the sufficient brain regions where Rut functions, in the rut ${ }^{1}$ mutant, to form visual pattern memory.

We currently do not know the exact role of rut in the fanshaped body and ellipsoid body; however, we can infer from previous studies on the larval neuromuscular junction (Zhong and Wu 1991; Cheung et al. 1999; Renger et al. 2000) that rut may mediate synaptic plasticity in these neurons. We assume that rutdependent synaptic plasticity may be lost in the rut ${ }^{1}$ or RNAi silencing flies, but only compromised in the rut $t^{2080}$ flies. Our results could be interpreted as that the loss of rut-dependent synaptic plasticity in either the fan-shaped body or ellipsoid body impaired visual pattern memory (Fig. 3A), but flies with a compromised fan-shaped body and a restored ellipsoid body, or a compromised ellipsoid body and a restored fan-shaped body, could form stable, wild-type memories (Figs. $1 \mathrm{C}$ and $2 \mathrm{~A}$ ). It seems that an operating range for the underlying neural circuit exists. Complete loss of rut-dependent synaptic plasticity in either the fan-shaped body or ellipsoid body moves the circuit out of the operating range, while restoring either of the compromised fan-shaped body or ellipsoid body can bring the circuit back into the operating range.

\section{Functional differentiation of the fan-shaped body and ellipsoid body}

It has been found in many insects including Drosophila that the central complex is involved in visual signal processing and motor control (Bausenwein et al. 1994; Martin et al. 1999; Strauss 2002; Vitzthum et al. 2002; Heinze and Homberg 2007; Ritzmann et al. 2008). However, the exact roles of the central complex substructures are not well understood. What we knew until now is that the F1 neurons are necessary for visual pattern memory for "contour orientation" and F5 neurons for "elevation," which raises the possibility that visual signals are processed in the fan-shaped body and distinct $F$ neurons are responsible for different visual pattern parameters. Recently, the R2/R4m neurons in the ellipsoid body were proved to be involved in ethanol sensitivity and tolerance (Urizar et al. 2007), and later in olfactory long-term memory consolidation (Wu et al. 2007). In our study, the R2/R4m neurons were found to be required for visual pattern memory for all tested parameters and thus may be parameter independent. However, the exact role of the R2/R4m neurons for visual pattern memory could not be determined yet.

\section{Concluding remarks}

Our studies indicated that Rut function in the central complex was crucial for Drosophila visual pattern memory; however, there might be some other Rut-independent neurons that also contribute to the neural circuit. Future work should focus on loss-offunction studies by blocking neural signaling in targeted subsystems. Furthermore, a temporal dissection of memory acquisition and retrieval would help us to understand how the different neuropils are involved. As the F and R neurons are all large field neurons that connect to other brain regions or other parts of the central complex (Hanesch et al. 1989), it is also crucial to identify the upstream and downstream neurons. Although the picture is far from complete, it seems that the central complex might be the major center for visual pattern memory. Studying such adaptive
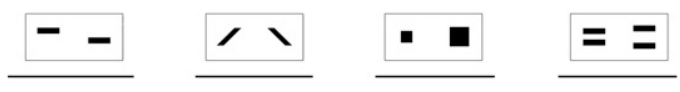

\section{A}

\section{$A$}
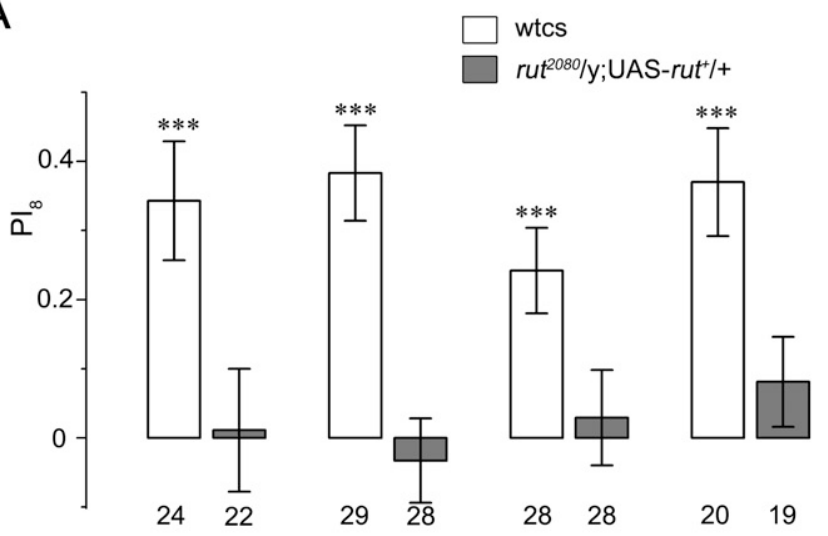

B

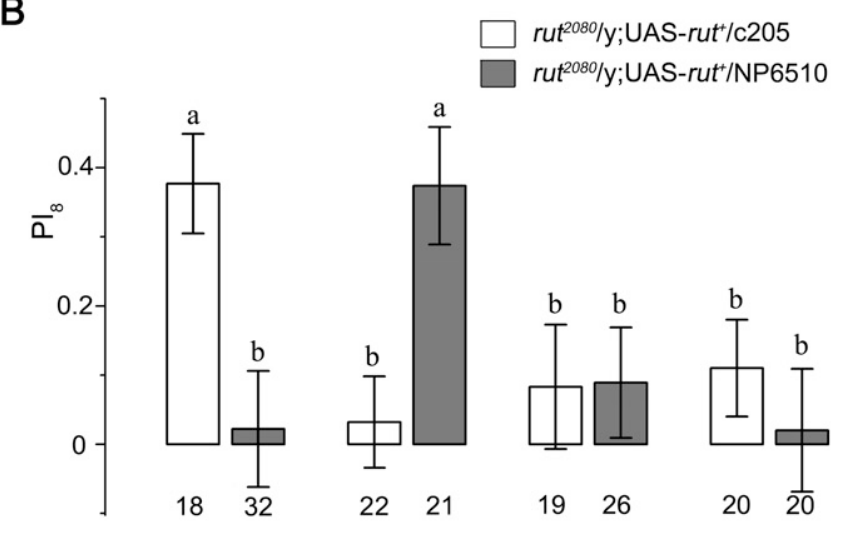

C
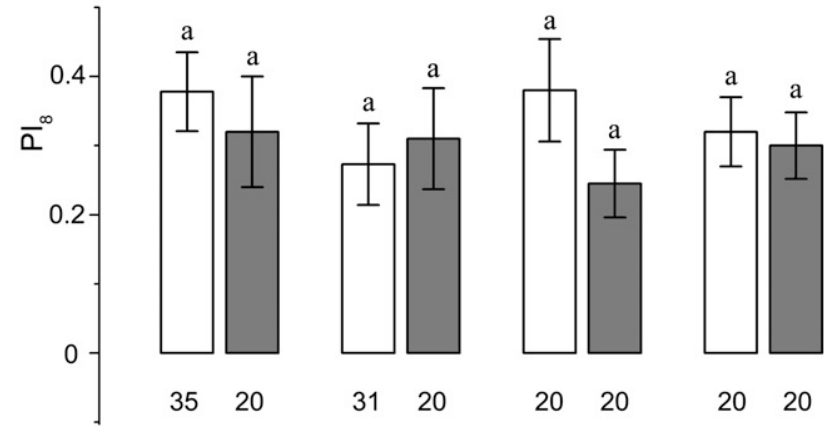

Figure 5. (Legend on next page) 
behaviors from a single gene to multiple types of neurons within a circuit is a challenging but indispensable step to unravel the neural basis of complex behaviors.

\section{Materials and Methods}

\section{Fly stocks}

All flies were maintained at $25^{\circ} \mathrm{C}$ (except for flies carrying tub$\mathrm{GAL}^{\mathrm{ts}}{ }^{\mathrm{t}}$ ) on standard corn meal/molasses medium (Guo et al. 1996) in a 12-h light/12-h dark cycle at $60 \%$ humidity. The rut $^{2080}$;UAS-rut ${ }^{+}$flies were previously described by Zars et al. (2000a,b). GAL4 lines c819, c232, c547, and c42 were kindly provided by U. Heberlein (University of California, San Francisco) The UAS-rut ${ }^{\mathrm{RNAi}}$ flies were from VDRC (Vienna Drosophila RNAi Center), and the $r u t^{1}$ flies were from the Bloomington stock center. All the GAL4 lines were outcrossed with $w^{1118}$ flies for six generations before use.

\section{Visual pattern memory assays}

Visual learning experiments were carried out in the Drosophila flight simulator (Fig. 1A). For apparatus, preparation, and handling of the flies, see the detailed description in Heisenberg and Wolf (1984). For the learning paradigm, procedure, and definition of performance index (PI), see Wolf and Heisenberg (1991) and Figure 1B. The reinforcer is an adjustable infrared laser (wavelength, $10.6 \mu \mathrm{m}$; power, $300 \mathrm{~mW}$ ) that is directed from above at the abdomen of the fly and controlled by the computer. The diameter of the laser spot is $0.4 \mathrm{~mm}$, and the laser beam is pulsed with 50-msec duration and 50-msec interval.

Flies carrying tub-GAL80 ${ }^{\text {ts }}$ were cultured at $19^{\circ} \mathrm{C}$. The experimental groups were shifted for $12 \mathrm{~h}$ to $30^{\circ} \mathrm{C}$ prior to behavioral tests, while the control groups without heat shock treatment were raised at $19^{\circ} \mathrm{C}$ through development, then kept at $25^{\circ} \mathrm{C}$ for adaptation just before behavioral tests. All behavioral experiments were performed at $25^{\circ} \mathrm{C}$.

For statistics, the two independent samples $t$-test was used to compare experimental groups with control ones, and the onesample $t$-test was carried out for the wild-type and mutant flies.

\section{Conditioned stimulus}

Upright and inverted T-shaped patterns were used for parameter "elevation" if not specially mentioned. T patterns measure $36^{\circ}$ vertically and $39^{\circ}$ horizontally; the bars of the Ts are $12^{\circ}$ wide. Four other patterns were used in this study (Fig. 5): (1) Parameter "elevation": horizontal bars $\left(40^{\circ} \times 10^{\circ}\right)$ placed at $10^{\circ}$ above and below the horizon; (2) parameter "contour orientation": bars of two different orientations $\left(-45^{\circ}\right.$ and $\left.45^{\circ}\right)$; (3) parameter "size": $20^{\circ} \times 20^{\circ}$ and $34^{\circ} \times 34^{\circ}$ squares; (4) parameter "vertical compactness": two horizontal bars $\left(40^{\circ} \times 10^{\circ}\right)$ with vertical distance of $20^{\circ}$ and $30^{\circ}$. All bars and squares were in black.

Figure 5. The $\mathrm{F}$ and $\mathrm{R}$ neurons function differentially for visual pattern memory. (A) Wild-type flies could form memories for four visual pattern parameters: elevation, contour orientation, size, and vertical compactness, but the rut $t^{2080}$ mutant flies with a copy of UAS-rut ${ }^{+}\left(r^{2080} / \mathrm{y} ; \mathrm{UAS}\right.$ $\mathrm{rut}^{+} /+$) did not. (B) Rescue in the fan-shaped body. Overexpression of $\mathrm{rut}^{+}$ in the F5 neurons (c205) specifically rescued memory for "elevation" but not the other three pattern parameters, while overexpression of rut targeted to the F1 neurons (NP6510) could only restore memory for "contour orientation" but none of the others. (C) Rescue in the ellipsoid body. Overexpression of rut in the R2/R4m neurons (c819) could restore memory for all parameters to wild-type levels, and the rescue effect could be repeated by another GAL4 driver line $c 42$, which also labels the R2/ R4m neurons. Numbers below the bars indicate numbers of flies. $\left.{ }^{* * *}\right) P<$ 0.001 (compared with zero, one sample t-test); (a) $P<0.05$ compared with flies without GAL4 driver $\left(\right.$ rut $^{2080} / \mathrm{y} ; \mathrm{UAS}-$ rut $\left.^{+} /+\right)$and $P>0.05$ compared with the wild-type flies. (b) $P>0.05$ compared with the rut $^{2080} / \mathrm{y}$; UAS-rut ${ }^{+} /+$flies. Comparisons were made between two groups using the same pattern parameter (two independent samples $t$-test). Error bars indicate SEM.
Flies' pattern discrimination abilities were measured as previously described (Liu et al. 2006). In brief, the orientation distribution of an individual fly during pretest was analyzed by Fourier analysis. The discrimination value $(D)$, which was used to evaluate a fly's pattern discrimination ability, was calculated as the rate of the amplitude of the $180^{\circ}$ component to the mean amplitude of the (near by) $120^{\circ}$ and $72^{\circ}$ components $\left(D=2 A_{180} /\right.$ $\left(A_{120}+A_{72}\right)$ subscripts refer to Fourier components; $A$ is amplitude). The chance value for $D$ is 1 . We evaluated the wild-type and $r u t^{2080}$ flies' ability to discriminate visual patterns and found that they both could discriminate all four pattern parameters used above (Table 1).

\section{Quantitative PCR}

Quantitative PCR (qPCR) was used in a standard way. In brief, 0.5 $\mu \mathrm{L}$ of cDNA mixtures prepared from the heads of 3- to 4-d-old flies was used as template and tested on a Chromo 4 system (MJ Research/Bio-Rad). For each fly strain, cDNA was obtained from four independent RNA preparations for repeating and averaging. The relative differences in rut mRNA expression levels were quantified by comparing their levels with standard curves, which were constructed using the corresponding recombinant plasmids, and normalized to the level of actin. One-way analysis of variance (ANOVA) was used for the statistical analysis of relative mRNA levels. The primers used for qPCR were as follows:

qPCR-actin upper, 5'-CAGGCGGTGCTTTCTCTCTA-3'; qPCR-actin lower, 5'-AGCTGTAACCGCGCTCAGTA-3'; qPCR-rut upper, 5'-TGTGATAAACGACGAGCTGA-3'; qPCR-rut lower, 5'-AGCAACGAGATGTTCTCCAG-3'.

\section{Immunohistochemistry}

Dissection of adult brains was performed in cold PBS (phosphate buffer saline) to remove the cuticle and connective tissues. After a brief wash in PBS, samples were fixed in freshly prepared $4 \%$ paraformaldehyde in PBS for $1 \mathrm{~h}$ at room temperature and rinsed $3 \times 15$ min each in 0.5\% PBT (PBS with $0.5 \%$ Triton X-100). Subsequently, samples were incubated with primary antibodies in $10 \%$ PNT (10\% normal goat serum in PBT) overnight at $4^{\circ} \mathrm{C}$, washed $3 \times 15$ min each with PBT, and then incubated in PNT containing secondary antibody. After three 15-min washes in PBT, brains were mounted in Vectashield (Vector Laboratories) and viewed with a Leica TCS SP5 confocal microscope. Raw confocal image sequences were imported into Image software to reconstruct 3D images. Modifications were made using Adobe Photoshop (Adobe Systems) to obtain optimal visual effects. Care was taken to ensure that brightness and contrast alterations were performed on the entire panel without losing any detail. Monoclonal mouse anti-nc82 (kindly provided by E. Buchner, University

Table 1. Visual pattern discrimination of the wild-type and rut $t^{2080}$ flies

\begin{tabular}{|c|c|c|}
\hline \multirow{2}{*}{ Visual stimuli } & \multicolumn{2}{|c|}{ Discrimination value $(D)$} \\
\hline & wtcs & rut $^{2080} / \mathrm{y} ;$ UAS-rut ${ }^{+} /+$ \\
\hline Four identical T patterns & $1.05 \pm 0.19(n=20)$ & N.D. \\
\hline Elevation ( $\mathrm{T}$ and inverted $\mathrm{T}$ ) & $2.43 \pm 0.46(n=44)$ & $2.60 \pm 0.42(n=45)$ \\
\hline Elevation (horizontal bars) & $3.58 \pm 0.86(n=24)$ & $2.44 \pm 0.41(n=22)$ \\
\hline Contour orientation & $3.18 \pm 0.57(n=29)$ & $2.32 \pm 0.42(n=28)$ \\
\hline Size & $2.39 \pm 0.41(n=28)$ & $3.59 \pm 0.68(n=28)$ \\
\hline Vertical compactness & $2.55 \pm 0.62(n=20)$ & $3.12 \pm 0.77(n=19)$ \\
\hline
\end{tabular}

For all the four tested visual pattern parameters, discrimination values $(D)$ of wild-type and rut ${ }^{2080}$ flies were not significantly different $(P>0.05$ for each pattern parameter, two independent samples $t$-test), although both significantly higher than the chance value $(D=1)(P<0.05$ for all comparisons, one sample $t$-test). As a control, in the experiment of wildtype flies with four identical T patterns, the discrimination values are not different from the chance value. 
of Wuerzburg, Germany) primary antibody was used at a dilution of 1:20. TRITC-conjugated anti-mouse secondary antibody (Jackson ImmunoResearch) was used at a 1:200 dilution.

\section{Acknowledgments}

We thank U. Heberlein and E. Buchner for providing fly stocks and antibody. We also thank J. Rister and the two anonymous reviewers for valuable comments on the manuscript. This work was supported by the "973 Program" (2005CB522804 and 2009CB918702), the National Natural Sciences Foundation of China (30621004, 30625022, and 30770682), and the Knowledge Innovation Program of the Chinese Academy of Sciences (KSCX2YW-R-28).

\section{References}

Akalal, D.B., Wilson, C.F., Zong, L., Tanaka, N.K., Ito, K., and Davis, R.L. 2006. Roles for Drosophila mushroom body neurons in olfactory learning and memory. Learn. Mem. 13: 659-668.

Bausenwein, B., Muller, N.R., and Heisenberg, M. 1994. Behaviordependent activity labeling in the central complex of Drosophila during controlled visual stimulation. J. Comp. Neurol. 340: 255-268.

Brand, A.H. and Perrimon, N. 1993. Targeted gene expression as a means of altering cell fates and generating dominant phenotypes. Development 118: $401-415$

Brembs, B. and Heisenberg, M. 2000. The operant and the classical in conditioned orientation of Drosophila melanogaster at the flight simulator. Learn. Mem. 7: 104-115.

Cheung, U.S., Shayan, A.J., Boulianne, G.L., and Atwood, H.L. 1999. Drosophila larval neuromuscular junction's responses to reduction of cAMP in the nervous system. J. Neurobiol. 40: 1-13.

Connolly, J.B., Roberts, I.J., Armstrong, J.D., Kaiser, K., Forte, M., Tully, T., and O'Kane, C.J. 1996. Associative learning disrupted by impaired Gs signaling in Drosophila mushroom bodies. Science 274: 2104-2107.

Dubnau, J., Grady, L., Kitamoto, T., and Tully, T. 2001. Disruption of neurotransmission in Drosophila mushroom body blocks retrieval but not acquisition of memory. Nature 411: 476-480.

Dudai, Y. and Zvi, S. 1984. Adenylate cyclase in the Drosophila memory mutant rutabaga displays an altered $\mathrm{Ca}^{2+}$ sensitivity. Neurosci. Lett. 47: 119-124.

Ernst, R. and Heisenberg, M. 1999. The memory template in Drosophila pattern vision at the flight simulator. Vision Res. 39: 3920-3933.

Guo, A., Li, L., Xia, S.Z., Feng, C.H., Wolf, R., and Heisenberg, M. 1996. Conditioned visual flight orientation in Drosophila: Dependence on age, practice, and diet. Learn. Mem. 3: 49-59.

Han, P.L., Levin, L.R., Reed, R.R., and Davis, R.L. 1992. Preferential expression of the Drosophila rutabaga gene in mushroom bodies, neural centers for learning in insects. Neuron 9: 619-627.

Hanesch, U., Fischbach, K.F., and Heisenberg, M. 1989. Neuronal architecture of the central complex in Drosophila melanogaster. Cell Tissue Res. 257: 343-366.

Heinze, S. and Homberg, U. 2007. Maplike representation of celestial $E$-vector orientations in the brain of an insect. Science 315: 995-997.

Heisenberg, M. and Wolf, R. 1984. Vision in Drosophila: Genetics of microbehavior. In Studies of brain function (ed. V. Braitenberg),Vol. 12. Springer, Berlin, Germany.

Heisenberg, M., Wolf, R., and Brembs, B. 2001. Flexibility in a single behavioral variable of Drosophila. Learn. Mem. 8: 1-10.

Levin, L.R., Han, P.L., Hwang, P.M., Feinstein, P.G., Davis, R.L., and Reed, R.R. 1992. The Drosophila learning and memory gene rutabaga encodes a $\mathrm{Ca}^{2+} /$ calmodulin-responsive adenylyl cyclase. Cell 68: 479489.

Liu, G., Seiler, H., Wen, A., Zars, T., Ito, K., Wolf, R., Heisenberg, M., and Liu, L. 2006. Distinct memory traces for two visual features in the Drosophila brain. Nature 439: 551-556.
Livingstone, M.S., Sziber, P.P., and Quinn, W.G. 1984. Loss of calcium/ calmodulin responsiveness in adenylate cyclase of rutabaga, a Drosophila learning mutant. Cell 37: 205-215.

Martin, J.R., Raabe, T., and Heisenberg, M. 1999. Central complex substructures are required for the maintenance of locomotor activity in Drosophila melanogaster. J. Comp. Physiol. 185: 277-288.

McGuire, S.E., Le, P.T., and Davis, R.L. 2001. The role of Drosophila mushroom body signaling in olfactory memory. Science 293: 13301333.

Neuser, K., Triphan, T., Mronz, M., Poeck, B., and Strauss, R. 2008. Analysis of a spatial orientation memory in Drosophila. Nature 453: 1244-1247.

Poeck, B., Triphan, T., Neuser, K., and Strauss, R. 2008. Locomotor control by the central complex in Drosophila an analysis of the tay bridge mutant. Dev. Neurobiol. 68: 1046-1058.

Popov, A.V., Peresleni, A.I., Ozerskii, P.V., Shchekanov, E.E., and SavvateevaPopova, E.V. 2005. The role of the flabellar and ellipsoid bodies of the central complex of the brain of Drosophila melanogaster in the control of courtship behavior and communicative sound production in males. Neurosci. Behav. Physiol. 35: 741-750.

Renger, J.J., Ueda, A., Atwood, H.L., Govind, C.K., and Wu, C.F. 2000. Role of cAMP cascade in synaptic stability and plasticity: Ultrastructural and physiological analyses of individual synaptic boutons in Drosophila memory mutants. J. Neurosci. 20: 3980-3992.

Renn, S.C., Armstrong, J.D., Yang, M., Wang, Z., An, X., Kaiser, K., and Taghert, P.H. 1999. Genetic analysis of the Drosophila ellipsoid body neuropil: Organization and development of the central complex. $J$. Neurobiol. 41: 189-207.

Ritzmann, R.E., Ridgel, A.L., and Pollack, A.J. 2008. Multi-unit recording of antennal mechano-sensitive units in the central complex of the cockroach, Blaberus discoidalis. J. Comp. Physiol. A Neuroethol. Sens. Neural Behav. Physiol. 194: 341-360.

Sakai, T. and Kitamoto, T. 2006. Differential roles of two major brain structures, mushroom bodies and central complex, for Drosophila male courtship behavior. J. Neurobiol. 66: 821-834.

Strauss, R. 2002. The central complex and the genetic dissection of locomotor behaviour. Curr. Opin. Neurobiol. 12: 633-638.

Strauss, R. and Heisenberg, M. 1993. A higher control center of locomotor behavior in the Drosophila brain. J. Neurosci. 13: 1852-1861.

Tang, S. and Guo, A. 2001. Choice behavior of Drosophila facing contradictory visual cues. Science 294: 1543-1547.

Thum, A.S., Jenett, A., Ito, K., Heisenberg, M., and Tanimoto, H. 2007. Multiple memory traces for olfactory reward learning in Drosophila. J. Neurosci. 27: 11132-11138.

Urizar, N.L., Yang, Z., Edenberg, H.J., and Davis, R.L. 2007. Drosophila homer is required in a small set of neurons including the ellipsoid body for normal ethanol sensitivity and tolerance. J. Neurosci. 27: 4541-4551.

Vitzthum, H., Muller, M., and Homberg, U. 2002. Neurons of the central complex of the locust Schistocerca gregaria are sensitive to polarized light. J. Neurosci. 22: 1114-1125.

Wang, Z., Pan, Y., Li, W., Jiang, H., Chatzimanolis, L., Chang, J., Gong, Z., and Liu, L. 2008. Visual pattern memory requires foraging function in the central complex of Drosophila. Learn. Mem. 15: 133-142.

Wolf, R. and Heisenberg, M. 1991. Basic organization of operant behavior as revealed in Drosophila flight orientation. J. Comp. Physiol. 169: 699-705.

Wu, C.L., Xia, S., Fu, T.F., Wang, H., Chen, Y.H., Leong, D., Chiang, A.S., and Tully, T. 2007. Specific requirement of NMDA receptors for long-term memory consolidation in Drosophila ellipsoid body. Nat. Neurosci. 10: 1578-1586.

Zars, T., Fischer, M., Schulz, R., and Heisenberg, M. 2000a. Localization of a short-term memory in Drosophila. Science 288: 672-675.

Zars, T., Wolf, R., Davis, R., and Heisenberg, M. 2000b. Tissue-specific expression of a type I adenylyl cyclase rescues the rutabaga mutant memory defect: In search of the engram. Learn. Mem. 7: 18-31.

Zhong, Y. and Wu, C.F. 1991. Altered synaptic plasticity in Drosophila memory mutants with a defective cyclic AMP cascade. Science 251: 198201.

Received January 12, 2009; accepted in revised form March 10, 2009. 


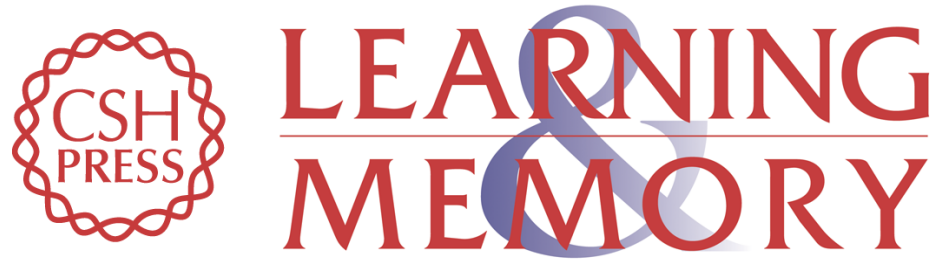

\section{Differential roles of the fan-shaped body and the ellipsoid body in Drosophila visual pattern memory}

Yufeng Pan, Yanqiong Zhou, Chao Guo, et al.

Learn. Mem. 2009, 16:

Access the most recent version at doi:10.1101//m.1331809

References This article cites 38 articles, 18 of which can be accessed free at: http://learnmem.cshlp.org/content/16/5/289.full.html\#ref-list-1

License

Email Alerting

Receive free email alerts when new articles cite this article - sign up in the box at the Service top right corner of the article or click here. 